\title{
Magnetic energy balance in the quiet Sun on supergranular spatial and temporal scales
}

25 portance in active regions as well as in the quiet Sun,

26 where they may trigger a chain of phenomena relevant

${ }_{27}$ for Space Weather. In this framework, a substantial contribution to the energy budget of the photosphere is carried by ubiquitous small-scale magnetic fields (magnetic elements, MEs) with characteristic size of the order 1 of - and smaller than - the spatial resolution (about 100 $2 \mathrm{~km}$ ) achievable by current instruments (see, e.g., Tru3 jillo Bueno et al. 2004; Bellot Rubio \& Orozco Suárez 4 2019). Several studies in the literature have pointed out 35 the key role played by MEs in storing energy in the quiet ${ }_{36}$ Sun and their capability to transfer it upward via, for 37 instance, magnetic reconnections (see, e.g., Chae 1999; 38 Viticchié et al. 2006; Rouppe van der Voort et al. 2016;

Corresponding author: Fabio Giannattasio fabio.giannattasio@ingv.it
39 Gošić et al. 2018; Bellot Rubio \& Orozco Suárez 2019, 40 and references therein) and/or magnetohydrodynamic 41 waves (see, e.g., Hahn \& Savin 2014; Stangalini et al. 42 2015; Jefferies et al. 2019; Rajaguru et al. 2019, and ref43 erences therein). However, the processes by which MEs ${ }_{44}$ emerge, evolve and organize in the quiet photosphere 45 are still not completely clear, despite the recent efforts 46 aimed to characterize their dynamics on a wide range 47 of spatial and temporal scales, from granular to super48 granular (see, e.g., Giannattasio et al. 2013, 2014b,a; 49 Abramenko 2017; Giannattasio et al. 2018; Bellot Ru50 bio \& Orozco Suárez 2019; Giannattasio et al. 2019, and 51 references therein).

In order to correctly estimate the amount of available ${ }_{3}$ energy in a given photospheric region it is necessary to 54 know the electric field, $\mathbf{E}$, and the current density, $\mathbf{J}$, as 55 well as the magnetic field, B. In fact, all these quanti56 ties allow to compute, for example, the Poynting flux, 57 the magnetic helicity (see, e.g., Démoulin \& Berger 2003; ${ }_{58}$ Schuck 2006; Kazachenko et al. 2014, 2015) and study 59 the evolution of currents and their coupling with electric ${ }_{60}$ and magnetic fields. In particular, the variation of the 
61 magnetic energy content in a volume of photospheric

62 plasma is linked to the work done by the field forces

63 on a distribution of charges via the Poynting's theorem,

64 which states that the rate of variation of the energy den-

65 sity equals the work done by the electric field plus the

${ }_{66}$ net rate of energy flux escaping the plasma volume el-

67 ement. More in detail, the rate of work done on the

68 surrounding plasma is expressed via the dot product,

${ }_{69} \mathbf{J} \cdot \mathbf{E}$, while the energy flux is described by the diver-

70 gence of the Poynting vector, $\mathbf{S}$. Thus, the interaction

71 between $\mathbf{E}, \mathbf{J}$ and $\mathbf{B}$ plays a fundamental role in the

72 energy balance of the photospheric plasma.

While the computation of the current density does not present criticalities once provided the vector magnetic field, and can be attained by invoking the Ampere's law, the computation of electric field is not trivial and has some aspects to pay attention to. Mainly two techniques 78 have been used in the past to compute the electric field: 79 the spectroscopy observation of the Stark effect (Wien 80 1916; Davis 1977; Jordan et al. 1980) and the use of the ${ }_{81}$ Ohm's law in the ideal MHD regime. While the former 82 method was recognized to be critically affected by the 83 low sensitivity of observations (Moran \& Foukal 1991); 84 the latter was improved by considering the component 85 of the Faraday's equation orthogonal to the magnetic 86 field so to obtain both velocity and electric field vectors 87 (Kusano et al. 2002; Welsch et al. 2004; Chae \& Sakurai 88 2008). Various refined techniques have been developed 89 to compute the electric field based on the Faraday's law 90 mixed with observational constraints (see, e.g., Fisher 91 et al. 2010; Kazachenko et al. 2014). These methods 92 are as accurate as complex, and require in input vector 93 magnetograms or full-Stokes data to perform spectropo94 larimetric inversions via suitable numerical procedures. ${ }_{95}$ These requirements imply the acceptance of trade-offs 96 in observations, as it is at present time still not pos97 sible to take advantage of robust vector magnetograms 98 (or full-Stokes data to be successfully inverted) at very 99 high spatial resolution and at the same time cover a wide 100 range of both spatial and temporal scales (from granular 101 to at least supergranular scales). However, when dealing 102 with observations targeted at the quiet Sun a reasonable 103 approximation for the photospheric electric field can be 104 still obtained also having only Line of Sight (LoS) mag105 netograms instead of full vector magnetograms as inputs 106 when averaging over the longest time scales available 107 (let's say of the order of typical time scale of supergran108 ulation). In this case the computation of electric field is 109 much simplified while, in contrast, none of the accurate 110 methods mentioned above is applicable to compute such 111 a "zeroth order" photospheric electric field.

112 As far as we know, the average properties of photo113 spheric electric field and current density in the quiet Sun 114 on supergranular spatial and temporal scales are still 115 not- or poorly- investigated, although they may play a 116 crucial role in the storage and dissipation of energy in 117 the quiet photosphere. In this work, for the first time 118 we provide an average zeroth-order description of the 119 properties of both the photospheric electric field and 120 current density in the quiet Sun on supergranular scales 121 and their connection with the magnetic energy budget 122 of the photosphere. We take advantage of an unprece123 dented data set consisting of a $\sim 24$ hr-long magne124 togram time series with high spatial resolution $(\simeq 0$ ". 3$)$ 125 targeted at the disk center and enclosing an entire su126 pergranule, whose linear size is about $\sim 50$ ". The results 127 obtained are discussed in the light of recent studies in 128 literature and may help to shed light on the mechanisms 129 that cause the variation of magnetic energy in the quiet 130 photosphere. The paper is organised as follows. In $\S 2$ 131 we describe the data set used and the approach by which 132 the physical quantities averaged on supergranular scales 133 are computed. $\S 3$ is devoted to the description of results 134 and their discussion in the light of the previous litera135 ture; while in $\S 4$ we summarize our findings and drive 136 to conclusions.

137

138

139 The data analyzed in this work were acquired by the 140 Hinode mission (Kosugi et al. 2007) on 2010 November ${ }_{141} 2$, and are part of the Hinode Operation Plan 151 enti142 tled "Flux replacement in the photospheric network and 143 internetwork". They consist of a magnetogram times se144 ries with 90s cadence starting at 08:00:42 UT, lasting for $145 \sim 24 \mathrm{hr}$ without interruption, and targeted at a quiet 146 Sun region in the disk center. Magnetograms were pro147 duced by using the spectral line $\mathrm{Na}$ I D at $589.6 \mathrm{~nm}$, 148 observed with the Narrowband Filter Imager (Tsuneta 149 et al. 2008) at two wavelengths at $\pm 160 \mathrm{~m} \AA$ from the line 150 center. Data were $2 \times 2$ binned to a pixel size of $0^{\prime \prime} .16$, 151 corresponding to $\simeq 116 \mathrm{~km}$ in the solar photosphere, and 152 a spatial resolution of $\simeq 0^{\prime \prime} .3$. The magneotogram noise ${ }_{153}$ is $\sigma \simeq 4 \mathrm{G}$ for single magnetograms, and was computed 154 as the rms of the signal in a sub-Field of View (sub-FoV) 155 free of magnetic field convolved with a $3 \times 3$ Gaussian 156 kernel. Magnetograms were co-aligned, trimmed to the 157 same FoV, which is $\simeq 51 \times 53 \mathrm{Mm}^{2}$ wide (corresponding 158 to $440 \times 455$ pixels $^{2}$ ), and filtered out for five minutes os159 cillations. Further details can be found in Gošić et al. ${ }_{160}$ (2014, 2016). 

174 several works on the same data set, and allowed to ob175 tain results reliable and consistent with previous obser176 vations and models (Orozco Suárez et al. 2012; Gošić 177 et al. 2014; Giannattasio et al. 2014b; Requerey et al. 178 2018; Chian et al. 2019). In particular, Orozco Suárez 179 et al. (2012) showed that the horizontal velocities ob180 tained in the same FoV with the FLCT technique origi181 nate radial velocity profiles within the supergranule that 182 are well fitted by the supergranular kinematic model in 183 Simon \& Weiss (1989); Simon et al. (2001). The mag184 netogram and horizontal velocity time series were then 185 averaged to recover the mean vertical magnetic- and hor186 izontal velocity- fields over $\sim 24 \mathrm{hr}$, which is comparable ${ }_{187}$ with the temporal scales characteristic of supergranula188 tion (Rast 2003; Del Moro et al. 2004). In Figure 1 we 190 show the mean magnetogram averaged over the whole 191 period of observation, $T$ ) of the FoV saturated between ${ }_{192}-300$ and $100 \mathrm{G}$. The boundaries of a supergranular cell 193 are clearly visible as magnetic field enhancements. The 194 green arrows represent the mean horizontal velocity field 195 as computed with the FLCT method (see also Figure 1a 196 in Giannattasio et al. 2014b).

${ }_{197}$ In the ideal case of very high magnetic Reynolds num198 bers such those in the solar photosphere (see, e.g., Parker 199 1963; Weiss 2001; Hirzberger 2002; Cattaneo et al. 2003; 200 Hood \& Hughes 2011; Rieutord et al. 2012) the conduc201 tivity diverges, and for the Ohm's law a finite current 202 density, $\mathbf{J}$, is possible only if

$$
\frac{\mathbf{J}}{\sigma}=\mathbf{E}+\frac{\mathbf{v}}{c} \times \mathbf{B}=0 \Rightarrow \mathbf{E}=-\frac{\mathbf{v}}{c} \times \mathbf{B},
$$

${ }_{203}$ where $\mathbf{E}, \mathbf{B}$, and $\mathbf{v}$ are the electric field, the magnetic 204 field and the plasma velocity, respectively, and we have 205 adopted cgs-Gaussian units. Let us consider the fol206 lowing geometry: the versor $\hat{\mathbf{z}}$ points upward along the 207 direction perpendicular to the photosphere, $\hat{\mathbf{y}}$ lays on 208 the photospheric plane and is directed toward the solar 209 North, and $\hat{\mathbf{x}}$ completes the orthonormal triad toward 210 the solar East. If we assume that the magnetic field av-

211 eraged on supergranular time scales $T$ is mainly vertical ${ }_{212}$ at photospheric heights (see the discussion in the next ${ }_{213}$ section) and in potential configuration (null helicity), 214 namely $\langle\mathbf{B}\rangle_{T} \simeq\left\langle B_{z}\right\rangle_{T} \hat{\mathbf{z}}$ with $\left\langle B_{x}\right\rangle_{T} \hat{\mathbf{x}}=\left\langle B_{y}\right\rangle_{T} \hat{\mathbf{y}}=0$, we 215 can estimate the average electric field as

$$
\langle\mathbf{E}\rangle_{T}=-\frac{1}{c}\langle\mathbf{v}\rangle_{T} \times\langle\mathbf{B}\rangle_{T} .
$$

${ }_{216}$ With this prescription the mean electric field reduces to

$$
\left\langle E_{x}\right\rangle_{T}=-\left\langle v_{y}\right\rangle_{T}\left\langle B_{z}\right\rangle_{T} / c \quad\left\langle E_{y}\right\rangle_{T}=\left\langle v_{x}\right\rangle_{T}\left\langle B_{z}\right\rangle_{T} / c \quad\left\langle E_{z}\right\rangle_{T}=0 .
$$

${ }_{217}$ In using the relations 3 the vertical magnetic field can ${ }_{218}$ be evaluated directly from the magnetogram time series 219 and the horizontal velocity field provided by the FLCT 220 technique. In the next section we will discuss the as221 sumption of vertical average magnetic field in the quiet 222 Sun and its evaluation via the magnetogram time series. 223 The current density that represents the source, at pho224 tospheric heights, of the observed magnetic field can be 225 inferred from the Ampere's law that in cgs-Gaussian 226 units reads

$$
\nabla \times \mathbf{B}=\frac{4 \pi}{c} \mathbf{J}
$$

227 228 229 230

$$
\left\langle J_{x}\right\rangle_{T}=\frac{c}{4 \pi} \frac{\partial\left\langle B_{z}\right\rangle_{T}}{\partial y} \quad\left\langle J_{y}\right\rangle_{T}=-\frac{c}{4 \pi} \frac{\partial\left\langle B_{z}\right\rangle_{T}}{\partial x} \quad\left\langle J_{z}\right\rangle_{T}=0 .
$$

231 The energy conservation in a plasma volume in pres232 ence of electric and magnetic fields is expressed by the ${ }_{233}$ Poynting theorem, which states the relation between the 234 energy density stored into an electromagnetic field, $u$, 235 the energy flux quantified by the Poynting vector, S, 236 and the work done by the fields on a charge distribu237 tion. In differential form and for the case $\sigma \rightarrow \infty$ it is 238 written

$$
-\frac{\partial u}{\partial t}=\nabla \cdot \mathbf{S}+\mathbf{J} \cdot \mathbf{E}
$$

239 where $u=B^{2} / 8 \pi$ is the (magnetic) field energy per 240 unit volume, $\mathbf{S}=\frac{c}{4 \pi} \mathbf{E} \times \mathbf{B}$ is the Poynting vector rep241 resenting the field energy flux, and $w \equiv \mathbf{J} \cdot \mathbf{E}$ is the ${ }_{242}$ rate of change of plasma mechanical energy per unit vol${ }_{243}$ ume. Thus, knowing the average photospheric electric 244 and magnetic fields and the current density on super245 graular scales, it is possible to estimate the right hand 246 side of Equation 6 and consequently the average rate 247 of change of field energy per unit volume on the super248 granular time scale $T$, namely $\langle\Delta U\rangle_{T}$. In particular, we 249 obtain

$$
\left\langle S_{x}\right\rangle_{T}=\frac{c}{4 \pi}\left\langle E_{y}\right\rangle_{T}\left\langle B_{z}\right\rangle_{T} \quad\left\langle S_{y}\right\rangle_{T}=-\frac{c}{4 \pi}\left\langle E_{x}\right\rangle_{T}\left\langle B_{z}\right\rangle_{T} \quad\left\langle S_{z}\right\rangle_{T}=0
$$




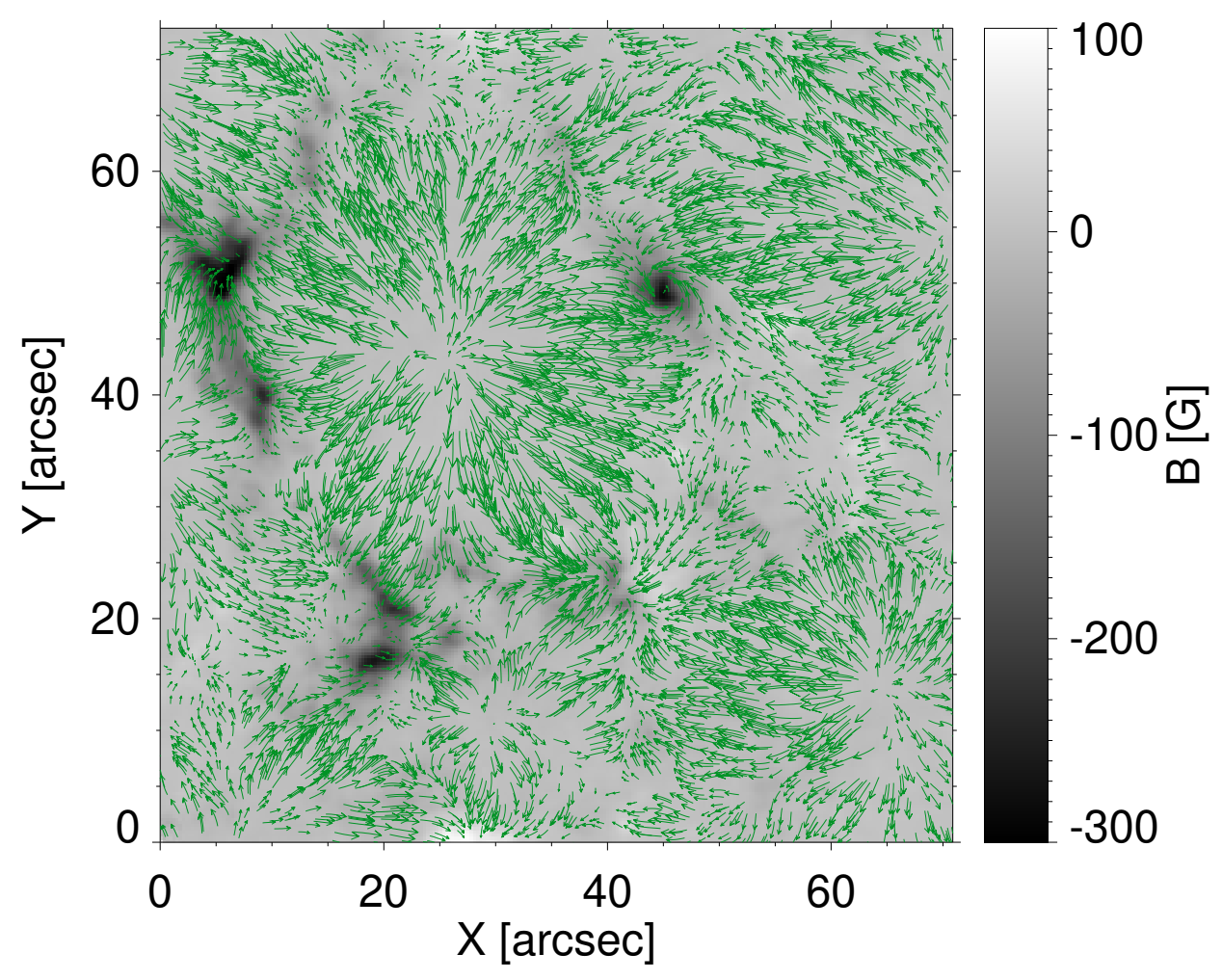

Figure 1. 24 hr-averaged magnetogram of the FoV saturated between -300 and 100 G. The boundaries of a supergranular cell are visible as enhancements of negative (black) field strengths. The green arrows represent the horizontal velocity field as computed with the FLCT method (see the text).

250 for $\langle\mathbf{S}\rangle_{T}$, which in this case is parallel to $\mathbf{v}$, and

$$
\langle w\rangle_{T}=\left\langle J_{x}\right\rangle_{T}\left\langle E_{x}\right\rangle_{T}+\left\langle J_{y}\right\rangle_{T}\left\langle E_{y}\right\rangle_{T}
$$

251 for $\langle w\rangle_{T}$, respectively.

253

254

${ }_{255}$ FoV under the hypothesis of very high Reynolds num256 bers and vertical magnetic field over the whole dura257 tion $T \simeq 24 \mathrm{hr}$ of observation by using Equations 3 . ${ }_{258}$ The computed mean electric field is shown in the up259 per panel of Figure 2. In that figure, the electric field 260 strength (saturated between $5 \cdot 10^{-5}$ and $3 \cdot 10^{-4}$ stat$261 \mathrm{volt} / \mathrm{cm}$ ) is represented in grey scale, while its direc262 tion is represented with golden arrows. As expected, ${ }_{263}$ the photospheric electric field is enhanced (and about ${ }_{264}$ one order of magnitude higher) in the boundaries of the 265 supergranular cell, where the magnetic network is lo266 cated (Giannattasio et al. 2014b), and the horizontal ${ }_{267}$ velocity is close to its maximum (Simon \& Weiss 1989; ${ }_{268}$ Orozco Suárez et al. 2012; Giannattasio et al. 2014b). ${ }_{269}$ Due to the mutual directions of $\mathbf{v}_{\mathbf{h}}$ and $\mathbf{B}$ the electric
270 field in the network regions either crosses the magnetic 271 field concentrations (see for example the region in the ${ }_{272}$ FoV at $X \in[0 " ; 10 "]$ and $\left.Y \in[35 " ; 55 "]\right)$, or departs 273 radially from them (see for example the region of the ${ }_{274}$ FoV at $X \in[40 " ; 50 "]$ and $\left.Y \in[45 " ; 55 "]\right)$. As we can 275 see in the horizontal velocity map shown in Figure 1 276 at the same locations, the former topology is associated 277 with a plasma motion parallel to the supergranular cell 278 boundary and towards increasing $Y$, while the latter is 279 associated with a counterclockwise whirling motion al280 ready detected in previous works (Bonet et al. 2008, 281 2010; Shelyag et al. 2011; Chian et al. 2019) with a char282 acteristic size of $\lesssim 5$ ", corresponding to $\lesssim 3.6 \mathrm{Mm}$ on 283 the photosphere.

${ }_{284}$ We evaluated the mean current density in the FoV, 285 namely $\langle\mathbf{J}\rangle_{T}$, by computing the components of Equa286 tion 5 . The results are shown in the lower panel of ${ }_{287}$ Figure 2, where the current density strength (saturated 288 between 2,000 and 10,000 statampere $/ \mathrm{cm}^{2}$ ) is repre289 sented in grey scale and its direction with white arrows. 290 As expected, the current density strength is enhanced 291 in correspondence with the magnetic network, and the 292 shape of the current density field is such to encircle the 293 magnetic field concentrations. It is interesting to notice 

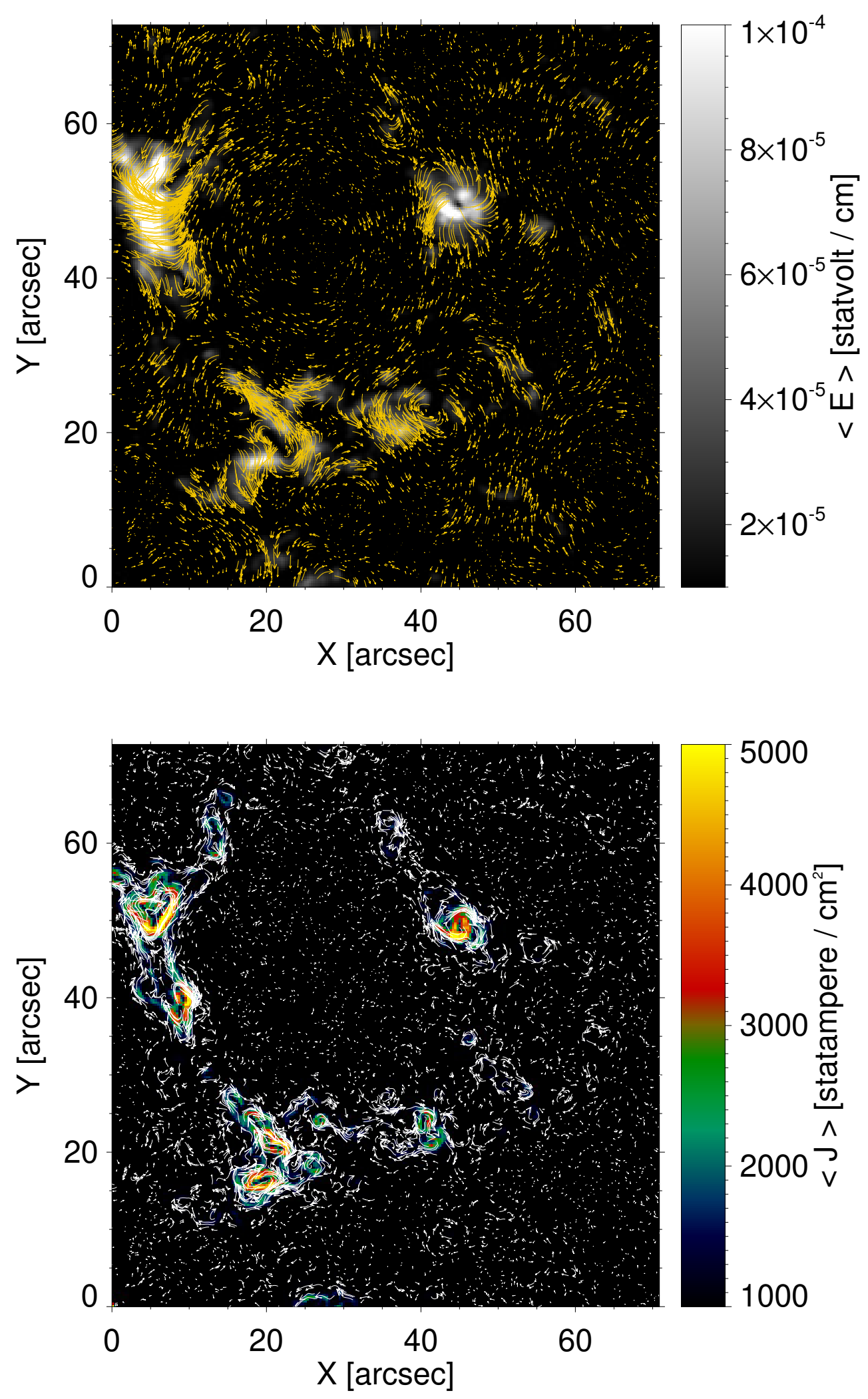

Figure 2. Upper panel: Mean electric field computed from Equations 3. The grey scale encodes the field strength, while the golden arrows show the direction of the electric field. Lower Panel: Mean current density computed from Equations 5 . The colour encode the strength, while the white arrows show the direction of the current density. 
294 the appearance of current density features that seem 295 to exhibit a hierarchy of vortexes, with the biggest sizes 296 around the strongest magnetic fields and a cascade down 297 to smaller-sized features in the surroundings. This is 298 visible especially in those regions at $Y \lesssim 40$ ". In most 299 models of turbulence, vortexes play a fundamental role, 300 as represent a mechanism able to continuously transfer 301 energy from the largest to the smallest scales, down to 302 the dissipation ones (Frisch 1995). However, the turbu303 lent nature of the current density features emerging in 304 the FoV used will be investigated in a future work.

305 We underline that the mean electric field and cur306 rent density were computed under the assumption that 307 the average horizontal component of the magnetic field 308 can be neglected compared to the vertical component, 309 which was estimated by considering the magnetogram 310 time series. This allowed us to use the relations 3 and 311 5. The question arises: Is this assumption reasonable? 312 The magnetic field inclination in the quiet Sun at pho313 tospheric heights is still a debated topic, and in the last 314 decades several works proposed controversial arguments 315 to assert the dominance of vertical fields over horizontal 316 ones or vice versa (see, e.g., Stenflo 2013a; Jafarzadeh 317 et al. 2014; Borrero et al. 2017; Kianfar et al. 2018; Bel318 lot Rubio \& Orozco Suárez 2019, and references therein). 319 No definitive conclusion to this debate was reached be320 cause of biases in observations and/or methods used to 321 investigate this topic (Jafarzadeh et al. 2014). How322 ever, in this work we take advantage of an unprecedented ${ }_{323}$ 24-hr long magnetogram time series containing a super324 granule. Over these spatial and temporal scales horizon325 tal field components, which typically take place in the 326 internetwork, are expected to average out, making their 327 contribution to the mean magnetic field negligible re328 spect to that of vertical fields. In fact, it is well known 329 that the magnetic field in the quiet Sun is ubiquitous 330 and quasi-isotropically distributed (Martin 1988; Meu331 nier et al. 1998; Lites 2002; Harvey et al. 2007; López 332 Ariste \& Sainz Dalda 2012). The histograms of mag333 netic field inclination and azimuth are consistent with 334 an isotropic distribution of transverse field associated 335 with the weakest fields and the presence of kilo-Gauss 336 fields that tend to be vertical (Stenflo 1982; Schüssler 337 1986; Orozco Suárez et al. 2007; Martínez González et al. 338 2008; Ishikawa \& Tsuneta 2009; Bommier et al. 2009; 339 Asensio Ramos 2009; Stenflo 2013b). An observational 340 evidence of the isotropic distribution of magnetic field 341 orientations is, for instance, the lack of Hanle rotation 342 when performing inversions of spectropolarimetric data ${ }_{343}$ (see, e.g., Bommier et al. 2005; Ishikawa et al. 2008; 344 Ishikawa \& Tsuneta 2009, 2010; Bellot Rubio \& Orozco 345 Suárez 2019, and references therein). In fact, in the last
346 decades the improvements in the inversion techniques al347 lowed to show that the azimuth PDFs are nearly flat in 348 the IN, indicating a random distribution of orientations 349 of the transverse field component.

350 Deviations from these values are only observed in 351 sunspots regions or for short time intervals. This is 352 not the case of the present work. Thus, on supergran353 ular scales many generations of shorter-living and arch354 shaped bipolar magnetic fields are expected to emerge 355 and evolve in the internetwork with randomly oriented 356 horizonthal components; while vertical fields are ex357 pected to survive, especially in the network and in the 358 nearby regions, where higher occurrences and longer 359 decorrelation times are observed (Welsch et al. 2012; Gi360 annattasio et al. 2018). Thus, the assumption that the 361 photospheric magnetic field in the quiet Sun is mainly 362 vertical on supegranular scales is reasonable. Under the 363 additional hypothesis that in the observed FoV the mag364 netic filling factors are $f f=1$ (Giannattasio et al. 2013), 365 the magnetogram time series used in this work provides 366 a reliable estimation for $\langle\mathbf{B}\rangle_{T} \simeq\left\langle B_{z}\right\rangle_{T} \hat{\mathbf{z}}$.

\section{3.2. Superdiffusion and the time scales of supergranulation}

369 Let us now consider the magneto-hydrodynamics 370 (MHD) induction equation describing the rate of change 371 of the magnetic field in a plasma volume. It reads

$$
\frac{\partial \mathbf{B}}{\partial t}=\nabla \times(\mathbf{v} \times \mathbf{B})+D_{m} \nabla^{2} \mathbf{B}
$$

372 where $D_{m}=c^{2} / 4 \pi \sigma$ is the magnetic diffusivity. As it is 373 well known, this equation describes the variation of the 374 magnetic field in terms of advection (first term in the 375 right-hand side, RHS) and diffusion (second term in the 376 RHS) by and across the plasma flow, respectively. Un377 der the ideal MHD hypothesis of magnetic field passively 378 transported by the plasma flow (see, e.g., Giannattasio 379 et al. 2013; Abramenko 2017) the diffusion term van380 ishes as $\sigma \rightarrow \infty$, and the magnetic field may be treated 381 like a passive scalar having no influence on the dynamics 382 of the surrounding plasma. It becomes a tracer helpful 383 to depict the behaviour of the underneath plasma veloc384 ity. By abuse of notation we can keep this term in order 385 to incorporate the (super-) diffusive properties of pho386 tospheric magnetic fields passively transported on supe387 granular scales by the underneath velocity field, which 388 is structured in a wide range of scales due to turbu389 lent convection (Berrilli et al. 2013, 2014; Giannattasio 390 et al. 2019), and substitute $D_{m}$ with the diffusivity $D$ 391 attributed to the spatially- and temporally- structured 392 plasma velocity field and computed, e.g., in Giannatta393 sio et al. (2013, 2014b). On the other hand, the ideal 
394 assumption is well fulfilled in the internetwork regions 395 (inside the supergranule) but is less valid in the network 396 regions, where the magnetic field may be strong (and 397 thick) enough to exert a not negligible magnetic pressure 398 on the surrounding plasma and resist the dragging of the 399 velocity field. In fact, while for MEs with field strength 400 below the equipartition value (Petrovay 1994; Giannat401 tasio et al. 2013) the drag force determines the motion ${ }_{402}$ and the ME transport is passive, in the opposite case ${ }_{403}$ of field strengths above the equipartition value (which 404 occurs in $\sim 4 \%$ of the magnetic fields in the FoV, Gian405 nattasio et al. 2013) MEs may move more independently 406 of the surrounding plasma velocity and be less effective 407 in passively tracing its behavior (Petrovay 1994). In this ${ }_{408}$ regime of, let's say, resistively transported magnetic field 409 the penetration of plasma across magnetic field may oc${ }_{410}$ cur, and tracking magnetic elements may probe a mix of ${ }_{411}$ dynamic properties from both the underneath velocity ${ }_{412}$ field and the magnetic field diffusing across plasma. It is ${ }_{413}$ thus interesting to estimate the magnitude of $D$ relative 414 to the pure advection term and the time scale of evolu415 tion of the magnetic field. To this aim, we can rewrite 416 Equation 9 in an "order-of-magnitude" form to highlight ${ }_{417}$ the characteristic spatial and temporal scales at work. ${ }_{418}$ The magnetic field variation time, $\tau$, on supergranular 419 scales, $l$, can be evaluated by rewriting Equation 9 as

$$
\frac{B}{\tau} \sim \frac{v B}{l}+D \frac{B}{l^{2}}
$$

420 On supergranular scales $l \sim 3 \cdot 10^{9} \mathrm{~cm}, v \sim 5 \cdot 10^{4} \mathrm{~cm} / \mathrm{s}$ ${ }_{421}$ (Giannattasio et al. 2014b), $D \sim 4 \cdot 10^{12} \mathrm{~cm}^{2} / \mathrm{s}$ at max422 imum (Giannattasio et al. 2013). The ratio between the 423 advection to diffusion terms is $l v / D \sim 25$ on supergran424 ular scales, meaning that the latter is at most $\sim 4 \%$ of 425 the former. From Equation 10 it follows that

$$
\tau \sim \frac{l^{2}}{l v+D} \sim \frac{10^{19}}{10^{14}+4 \cdot 10^{12}} \sim 10^{5} s,
$$

426 or, in other words, $\tau \sim 28 \mathrm{hr}$. Firstly, it emerges that ${ }_{427}$ even by considering the maximum superdiffusive term ${ }_{428} D$ measured by Giannattasio et al. (2013) in the net429 work regions (i.e. in resistively transported magnetic 430 field regime), it is negligible in the computation of $\tau$ as 431 it gives a contribution of at most $4 \%$ of that from the ${ }_{432}$ advection term and represents only a small correction. ${ }_{433}$ This is, again, consistent with the hypothesis of diverg434 ing conductivity in the framework of ideal MHD, which 435 remains a good approximation even within the network, 436 where whatever the dynamics is, it deviates only slightly ${ }_{437}(\sim 4 \%)$ from the ideal case $D=0$. Secondly, due to this ${ }_{438}$ small $D / l v$ ratio, the time scale of magnetic field varia439 tion on supergranular scales can be computed neglecting
440 diffusion and is fully consistent with the typical lifetime 441 of supergranules.

\section{3.3. The energy balance and the time scales of energy 443 exchange}

444 In a recent work, Giannattasio et al. (2018) showed 445 that the decorrelation time of magnetic field in the same ${ }_{446} \mathrm{FoV}, t_{D}$, which is the time after which the autocorrela447 tion function of pixel-by-pixel magnetogram signal drops 448 to $1 / e$, is between $\sim 0.5$ and $\sim 4 \mathrm{hr}$ in the supergran449 ular boundaries. This means that the magnetic field 450 on supergranular scales decorrelates well before the de451 cay time $\tau$, and it is not sufficient to consider only the 452 evolution of the magnetic field due to the underneath 453 velocity field in order to explain the much faster decor${ }_{454}$ relation $t_{d}<\tau$. We have to consider also the energy 455 that the magnetised plasma exchanges with the sur456 roundings. In fact, both the incoming and outgoing 457 energy flows to/from any plasma volume element may 458 increase/decrease the local energy budget and result in 459 a modification of the magnetic flux content and its con460 sequent decorrelation. Such a local energetic balance is 461 described by the Poynting Theorem (Equation 6). The ${ }_{462}$ simultaneous knowledge of $\langle\mathbf{E}\rangle_{T},\langle\mathbf{J}\rangle_{T}$ and $\langle B z\rangle_{T}$ al${ }_{463}$ lowed us to estimate the RHS of Equation 6 averaged 464 on supergranular time scales. In that equation, the first 465 term in RHS characterizes the energy flux that can be 466 eventually carried by an electromagnetic field and prop${ }_{467}$ agate through a plasma volume element, i.e. the Poynt${ }_{468}$ ing flux, and $\operatorname{div}(\mathbf{S})>0$ corresponds to an outflow of en469 ergy from the plasma volume element, while $\operatorname{div}(\mathbf{S})<0$ 470 corresponds to an inflow of energy in the same volume 471 element. The second term of RHS, $w$, has the dimension 472 of a power per unit volume and provides an estimate of ${ }_{473}$ the rate at which the Lorentz force does work on the 474 surrounding plasma causing an increase or decrease of 475 the magnetic energy, $u$. In fact, by dotting the Lorentz 476 force per unit volume, namely $\mathbf{f}=\rho \mathbf{E}+\mathbf{J} \times \mathbf{B}$, by the 477 plasma velocity $\mathbf{v}$ we obtain

$$
\mathbf{f} \cdot \mathbf{v}=\mathbf{J} \cdot \mathbf{E}=w
$$

${ }_{478}$ being the magnetic $\mathbf{J} \times \mathbf{B}$ term orthogonal to the veloc479 ity $\mathbf{~}$. Thus, only the electric field term of the Lorentz 480 force does work on the surrounding plasma. In par481 ticular, a positive variation, $w>0$, corresponds to a 482 mechanical work done by the fields on the surround${ }_{483}$ ing plasma, the more aligned currents and the electric 484 field are, the greater the amount of energy transferred 485 to the surrounding plasma. On the contrary, a negative ${ }_{486}$ variation, $w<0$, corresponds to an increase of internal ${ }_{487}$ energy as the Lorentz force does work in the opposite di${ }_{488}$ rection, being directed against the electric field from the 
489 surrounding plasma to the plasma volume element under 490 consideration. The critical values $\operatorname{div}(\mathbf{S})=0$ and $w=0$ 491 correspond, respectively, to a balance between the in492 flowing/outflowing electromagnetic energy through the 493 volume element and a null exchange of energy with the ${ }_{498}$ surrounding plasma. In the upper panel of Figure 3 we 496 show the time-averaged rate of change of mechanical en497 ergy per unit volume, $w$, saturated between -0.2 and 0.2 ${ }_{498} \mathrm{erg} \mathrm{cm}^{-3} \mathrm{~s}^{-1}$ and attributed to the Lorenz force acting 499 on current density via the electric field. The quantity $w$ 500 ranges between -0.92 and $0.27 \mathrm{erg} / \mathrm{cm}^{3} \mathrm{~s}$. In correspon501 dence of the supergranular boundaries there is an en502 hancement of this quantity in absolute value, such that 503 the appearing features are quite symmetrically divided 504 into adjacent sub-regions with opposite sign (blue/red 505 for negative/positive, respectively). This is consistent 506 with the coexistence of nearby regions where, on aver507 age, energy is lost (gained) due to the positive (negative) 508 work done by the Lorenz force per unit volume, the sign 509 being driven by the mutual directions of vectors $\mathbf{J}$ and 510 E. In these promiscuous regions the observed transition 511 between positive and negative values of $w$ occurs in the 512 center, where $w=0$. The only way to satisfy this condi513 tion is that the current density and the electric field are 514 mutually orthogonal, as on average neither the former 515 nor the latter are null.

${ }_{516}$ In the lower panel of Figure 3 we show the time517 averaged variation of the divergence of the Poynting 518 vector, $\operatorname{div}(\mathbf{S})$ that should be associated with an elec519 tromagnetic energy flow saturated between -0.8 and ${ }_{520} 0.8 \mathrm{erg} \mathrm{cm}^{-3} \mathrm{~s}^{-1}$. The quantity $\operatorname{div}(\mathbf{S})$ ranges between ${ }_{521}-1.23$ and $1.59 \mathrm{erg} / \mathrm{cm}^{3} \mathrm{~s}$. Also in this case, in corre522 spondence of the supergranular boundaries there is an 523 enhancement of this quantity in absolute value, which ${ }_{524}$ appears to be symmetrically divided into adjacent sub525 regions with opposite sign. This implies the coexis526 tence of nearby regions where, on average, energy is lost ${ }_{527}$ (gained) due to the positive (negative) energy flux, the ${ }_{528}$ sign being driven by the mutual directions of vectors $\mathbf{B}$ ${ }_{529}$ and $\mathbf{E}$. In these promiscuous regions the observed tran530 sition between positive and negative values of $\operatorname{div}(\mathbf{S})$ 531 occurs, again, in the centre, where $\operatorname{div}(\mathbf{S})=0$. The 532 only way to satisfy this condition is that the magnetic 533 and electric fields are parallel, as on average neither the ${ }_{534}$ former nor the latter are null. We note that the two 535 RHS terms in Equation 6 are of the same order of mag536 nitude, thus both contribute with the same weight to 537 the estimation of the energy density variation averaged 538 on supergranular scales, namely $\langle\Delta u\rangle_{T}$.

${ }_{539}$ The timescale, $\tau^{*}$, associated with the energy varia540 tion of a plasma volume element on supergranular scales
${ }_{541}$ can be computed by rewriting Equation 6 as follows:

$$
\frac{u}{\tau^{*}} \sim J E+\frac{S}{l}
$$

${ }_{542}$ By assuming $u \simeq B^{2} / 8 \pi$ with $B \sim 300 \mathrm{G}$ as a typical 543 value for the magnetic field in the FoV (Giannattasio 544 et al. 2013), considering the supergranular length scale ${ }_{545} l \sim 3 \cdot 10^{9} \mathrm{~cm}$ like did above, and once computed the ${ }_{546}$ Poynting vector $S \sim 10^{8} \mathrm{erg} / \mathrm{cm}^{2}$ s we evaluated the time 547 scale of energy exchange

$$
\tau^{*} \sim \frac{B^{2} l}{8 \pi(J E l+S)} \sim \frac{9 \cdot 10^{4} \cdot 3 \cdot 10^{9}}{8 \cdot 3 \cdot\left(5 \cdot 10^{3} \cdot 10^{-4} \cdot 3 \cdot 10^{9}+10^{8}\right)} \sim 10^{4} s,
$$

548 which corresponds to $\tau^{*} \sim 2.8 \mathrm{hr}$. This time scale is 549 of the same order of magnitude of the magnetic field 550 decorrelation times observed in the same FoV by Gi551 annattasio et al. (2018). This suggests that the energy 552 balance due to the interaction of plasma with both pho553 tospheric electric and magnetic fields on supergranular 554 scales plays a crucial role in modifying the magnetic pat555 terns that characterize the photospheric supergranula556 tion. In order to show this, in Figure 4 we show the av557 eraged LHS of Equation 6, $\langle\Delta u\rangle$ on supergranular scales 558 saturated between -1.5 and $1.5 \mathrm{erg} / \mathrm{cm}^{3} \mathrm{~s}$. The quantity ${ }_{559}\langle\Delta u\rangle$ ranges between -1.76 and $2.08 \mathrm{erg} / \mathrm{cm}^{3} \mathrm{~s}$. In that 560 figure, we superposed in green contour plots of the mag561 netic decorrelation times $t_{D}>120$ min computed in 562 Giannattasio et al. (2018). As we can see, the longer $t_{D}$ 563 times occur mostly where $\langle\Delta u\rangle \geq 0$, i.e. where the aver564 age energy variation is null or moderately positive. This 565 means that magnetic field decorrelates at longer times 566 mainly where the energy variation is null (in a station567 ary situation), as we may expect, or the energy slightly 568 increases, as this energy supply is effective in contrasting 569 the field decay and the consequent decorrelation. The 570 only exception is represented by the vortex motion ob571 served in the region of the FoV at $X \in[40 " ; 50 "]$ and ${ }_{572} Y \in[45 " ; 55 "]$, within which we have basically $\langle\Delta u\rangle \sim 0$, 573 and only a few very small sub-areas in the centre are as574 sociated with longer $t_{D}$ times. As found by Giannattasio 575 et al. (2018) this region is characterised by a very high 576 magnetic field occurrence (near $100 \%$ ) and $40 \lesssim t_{D} \lesssim 50$ $577 \mathrm{~min}$, which is probably due to the presence of different 578 and tightly packed magnetic elements moving in a very 579 restricted area. Thus, the lack of magnetic fields with 580 long $t_{D}$ times in this region with $\langle\Delta u\rangle=0$ is consistent 581 with the presence of an intense vortex that may act as an 582 attractor constraining the dynamics of the nearby mag583 netic elements to evolve in a very restricted area and ${ }_{588}$ causing these magnetic elements to pile up there. We 586 can interpret these results by depicting the following ${ }_{587}$ simple scenario. Turbulent convection produces mag- 

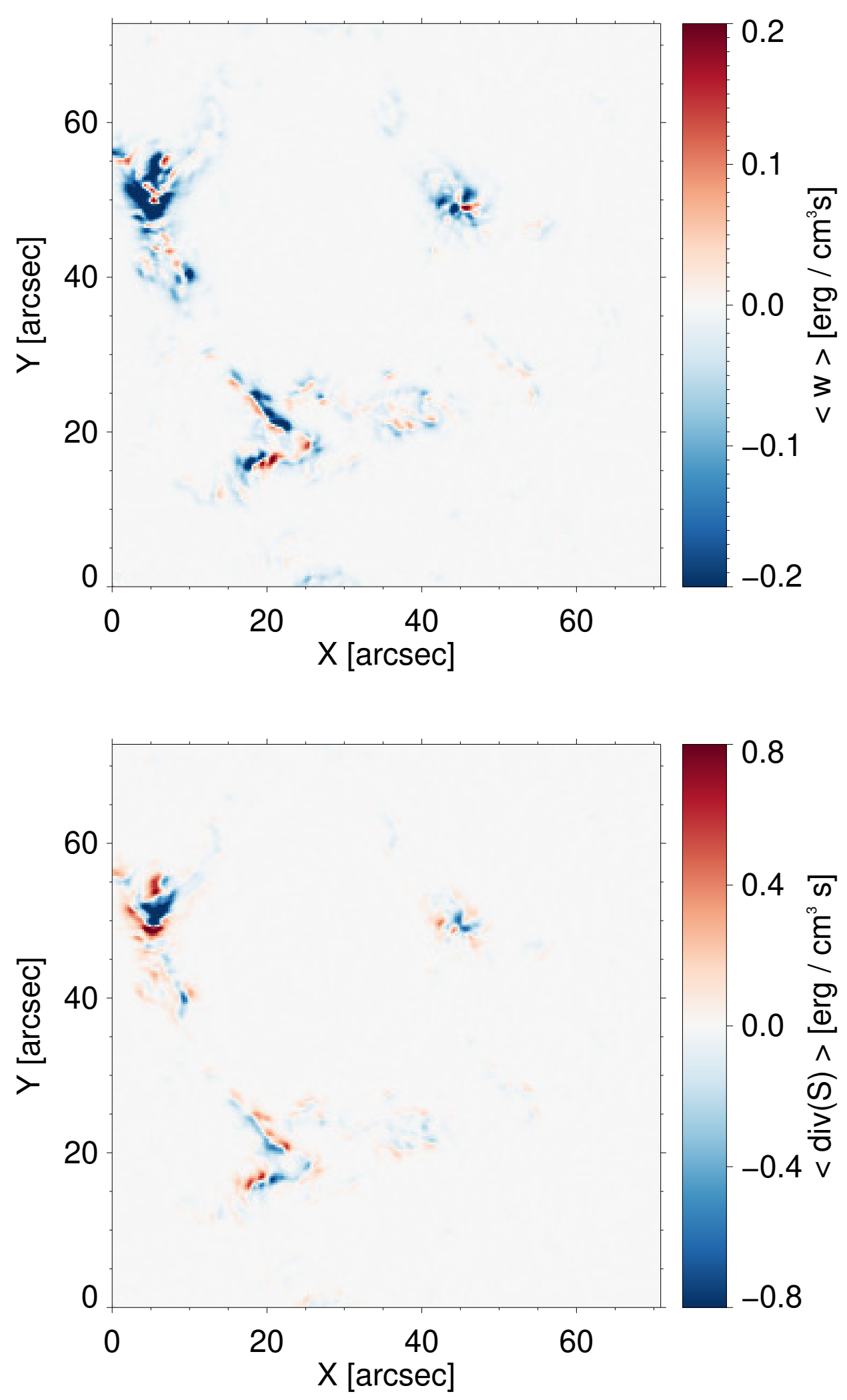

Figure 3. Upper panel: Mean energy variation rate, $w$, due to the Lorentz force. Lower panel: Mean divergence of the Poynting vector, i.e. the electromagnetic field energy flux available in the plasma volume element.

588 netic fields and drives their motion in the solar photo- $\quad 589$ sphere at all scales, from sub-granular to supergranular. 


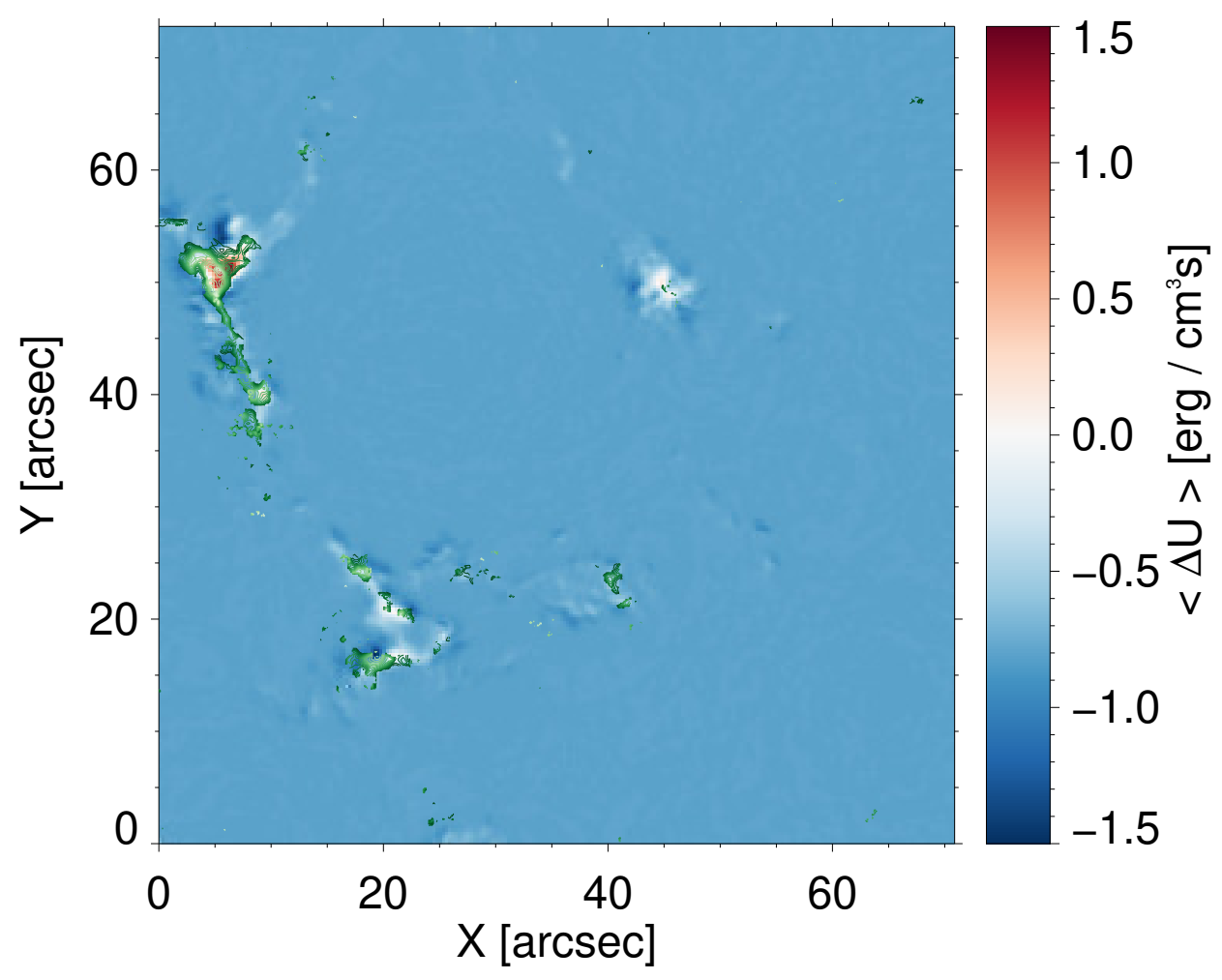

Figure 4. Mean energy variation rate, $\langle\Delta u\rangle$ saturated between -1.5 and $1.5 \mathrm{erg} / \mathrm{cm}^{3} \mathrm{~s}$. The superposed green lines are contour plots of the magnetic decorrelation times $t_{D}>120$ min computed in Giannattasio et al. (2018) (see the text).

615 must be sustained by both the enhancement of currents ${ }_{616}$ and an energy flux coming from the nearby regions, in 617 form, for example, of turbulent transport.

\section{SUMMARY AND CONCLUSIONS}

${ }_{619}$ Magnetic elements (MEs) are ubiquitous in the quiet ${ }_{620}$ photosphere. Studying their dynamic properties may 621 help to shed light on both the mechanisms of storage and 622 transfer of energy to the upper atmospheric layers, and ${ }_{623}$ the dynamic properties of the photospheric turbulent ${ }_{624}$ velocity field under the hypothesis that MEs are pas625 sively transported by the plasma flow. The knowledge ${ }_{626}$ of the electric field and current density together with the 627 magnetic field, allows to estimate the energy balance in ${ }_{628}$ the photosphere via the Poynting theorem, which links 629 the rate of variation of the energy density in a plasma 630 volume element with the work done by the electric field 631 on the surrounding plasma and the energy flux flowing 632 through the volume element. However, the computation 633 of local electric field at any time requires the knowledge, 634 for example, of the vector magnetic field, which can be 635 obtained only via the inversion of spectropolarimetric ${ }_{636}$ (SP) full-Stokes data. On the other hand, it is not pos637 sible to acquire long $\mathrm{SP}$ data targeted at large FoVs ${ }_{638}$ with fast cadence and high spectropolarimetric sensi- 
639 tivity, since this experimental setup has the result of 640 reducing the number of spectral points sampled, which ${ }_{641}$ affects the goodness of results, and vice versa. Despite of 642 this, we can still obtain a reasonable approximation for 643 the electric field averaged on supergranular scales by us644 ing only magnetogram time series instead of full-Stokes 645 data. Our findings may be itemized as follows:

1. For the first time we provided average photospheric electric field and current density in the quiet Sun on supergranular scales by using a $\sim 24$ hr-long magnetogram time series enclosing an entire supergranule;

2. By applying the Poynting theorem we computed the average rate of change of field energy per unit volume on supergranular scales, $\langle\Delta u\rangle$, and found that the timescale associated with the energy variation is consistent with the magnetic field decorrelation times, $t_{D}$, in the same FoV retrieved in Giannattasio et al. (2018);

3. The longer $t_{D}$ times are co-spatial with the regions where $\langle\Delta u\rangle \geq 0$, indicating that the energy supply effectively balances the magnetic field and energy decay.
${ }_{662}$ We regard that this study could represent a turning ${ }_{663}$ point for the exploitation of long magnetogram time se664 ries to investigate more comprehensively the energy bal665 ance at large and long scales. Due to the huge amount 666 of magnetic flux emerging in the quiet Sun, this energy ${ }_{667}$ should give a fundamental contribution to sustain the 668 upper atmospheric layers.

\section{ACKNOWLEDGMENTS}

This work is supported by tne Italian MIUR-PRIN grant 2017APKP7T on Circumterrestrial Environment: Impact of Sun-Earth Interaction. FG is grateful to M. Gošić and L. Bellot Rubio for providing the data here analyzed. This paper is based on data acquired in the framework of the Hinode Operation Plan 151 entitled "Flux replacement in the network and internetwork". Hinode is a Japanese mission developed and launched by ISAS/JAXA, collaborating with NAOJ as a domestic partner, NASA and STFC (UK) as international partners. Scientific operation of the Hinode mission is conducted by the Hinode science team organized at ISAS/JAXA. This team mainly consists of scientists from institutes in the partner countries. Support for the post-launch operation is provided by JAXA and NAOJ (Japan), STFC (U.K.), NASA, ESA, and NSC (Norway).

\section{REFERENCES}

\footnotetext{
Abramenko, V. I. 2017, MNRAS, 471, 3871, doi: 10.1093/mnras/stx1880

Asensio Ramos, A. 2009, ApJ, 701, 1032, doi: 10.1088/0004-637X/701/2/1032

Bellot Rubio, L., \& Orozco Suárez, D. 2019, Living Reviews in Solar Physics, 16, 1, doi: 10.1007/s41116-018-0017-1

Berrilli, F., Scardigli, S., \& Del Moro, D. 2014, A\&A, 568, A102, doi: 10.1051/0004-6361/201424026

Berrilli, F., Scardigli, S., \& Giordano, S. 2013, SoPh, 282, 379, doi: 10.1007/s11207-012-0179-2

Bommier, V., Derouich, M., Landi Degl'Innocenti, E., Molodij, G., \& Sahal-Bréchot, S. 2005, A\&A, 432, 295, doi: 10.1051/0004-6361:20035773

Bommier, V., Martínez González, M., Bianda, M., et al. 2009, A\&A, 506, 1415, doi: 10.1051/0004-6361/200811373

Bonet, J. A., Márquez, I., Sánchez Almeida, J., Cabello, I., \& Domingo, V. 2008, ApJL, 687, L131, doi: 10.1086/593329

Bonet, J. A., Márquez, I., Sánchez Almeida, J., et al. 2010, ApJL, 723, L139, doi: 10.1088/2041-8205/723/2/L139
}

689 Borrero, J. M., Jafarzadeh, S., Schüssler, M., \& Solanki,

690 S. K. 2017, SSRv, 210, 275,

691 doi: $10.1007 / \mathrm{s} 11214-015-0204-5$

692 Cattaneo, F., Emonet, T., \& Weiss, N. 2003, ApJ, 588,

693 1183, doi: 10.1086/374313

${ }_{694}$ Chae, J. 1999, in Astronomical Society of the Pacific

695 Conference Series, Vol. 183, High Resolution Solar

696 Physics: Theory, Observations, and Techniques, ed. T. R.

${ }_{697}$ Rimmele, K. S. Balasubramaniam, \& R. R. Radick, 375

${ }_{698}$ Chae, J., \& Sakurai, T. 2008, ApJ, 689, 593,

699 doi: $10.1086 / 592761$

700 Chian, A. C. L., Silva, S. S. A., Rempel, E. L., et al. 2019,

701 MNRAS, 488, 3076, doi: 10.1093/mnras/stz1909

702 Davis, W. D. 1977, SoPh, 54, 139, doi: 10.1007/BF00146430

703 Del Moro, D., Berrilli, F., Duvall, T. L., J., \& Kosovichev,

704 A. G. 2004, SoPh, 221, 23,

705 doi: 10.1023/B:SOLA.0000033363.15641.8f

706 Démoulin, P., \& Berger, M. A. 2003, SoPh, 215, 203,

707 doi: 10.1023/A:1025679813955 
708 Fisher, G. H., \& Welsch, B. 2007, in American

712 Fisher, G. H., \& Welsch, B. T. 2008, in Astronomical

737 Gošić, M., de la Cruz Rodríguez, J., De Pontieu, B., et al.

738 2018, ApJ, 857, 48, doi: 10.3847/1538-4357/aab1f0

739 Hahn, M., \& Savin, D. W. 2014, ApJ, 795, 111,

740 doi: 10.1088/0004-637X/795/2/111

741 Harvey, J. W., Branston, D., Henney, C. J., Keller, C. U., 742 \& SOLIS and GONG Teams. 2007, ApJL, 659, L177,

743 doi: $10.1086 / 518036$

744 Hirzberger, J. 2002, A\&A, 392, 1105,

745 doi: 10.1051/0004-6361:20020902

746 Hood, A. W., \& Hughes, D. W. 2011, Physics of the Earth

747 and Planetary Interiors, 187, 78,

748 doi: $10.1016 /$ j.pepi.2011.04.010

749 Ishikawa, R., \& Tsuneta, S. 2009, A\&A, 495, 607,

750 doi: 10.1051/0004-6361:200810636

751 -. 2010, ApJL, 718, L171,

752 doi: $10.1088 / 2041-8205 / 718 / 2 / \mathrm{L} 171$

753 Ishikawa, R., Tsuneta, S., Ichimoto, K., et al. 2008, A\&A,

754 481, L25, doi: 10.1051/0004-6361:20079022

755 Jafarzadeh, S., Solanki, S. K., Lagg, A., et al. 2014, A\&A,
757 Jefferies, S. M., Fleck, B., Murphy, N., \& Berrilli, F. 2019,

758 ApJL, 884, L8, doi: 10.3847/2041-8213/ab4719

759 Jordan, C., Bartoe, J. D. F., \& Brueckner, G. E. 1980,

760 ApJ, 240, 702, doi: 10.1086/158278

761 Kazachenko, M. D., Fisher, G. H., \& Welsch, B. T. 2014,

762 ApJ, 795, 17, doi: 10.1088/0004-637X/795/1/17

763 Kazachenko, M. D., Fisher, G. H., Welsch, B. T., Liu, Y.,

764 \& Sun, X. 2015, ApJ, 811, 16,

765 doi: $10.1088 / 0004-637 \mathrm{X} / 811 / 1 / 16$

766 Kianfar, S., Jafarzadeh, S., Mirtorabi, M. T., \&

767 Riethmüller, T. L. 2018, SoPh, 293, 123,

768 doi: $10.1007 / \mathrm{s} 11207-018-1341-2$

769 Kosugi, T., Matsuzaki, K., Sakao, T., et al. 2007, SoPh,

770 243, 3, doi: 10.1007/s11207-007-9014-6

771 Kusano, K., Maeshiro, T., Yokoyama, T., \& Sakurai, T.

$772 \quad$ 2002, ApJ, 577, 501, doi: 10.1086/342171

773 Lites, B. W. 2002, ApJ, 573, 431, doi: 10.1086/340120

774 López Ariste, A., \& Sainz Dalda, A. 2012, A\&A, 540, A66,

775 doi: 10.1051/0004-6361/201118191

776 Martin, S. F. 1988, SoPh, 117, 243,

777 doi: 10.1007/BF00147246

778 Martínez González, M. J., Asensio Ramos, A., López

779 Ariste, A., \& Manso Sainz, R. 2008, A\&A, 479, 229,

780 doi: 10.1051/0004-6361:20078500

781 Meunier, N., Solanki, S. K., \& Livingston, W. C. 1998,

$782 \quad$ A\&A, 331, 771

783 Moran, T., \& Foukal, P. 1991, SoPh, 135, 179,

784 doi: $10.1007 / \mathrm{BF} 00146705$

785 Orozco Suárez, D., Katsukawa, Y., \& Bellot Rubio, L. R.

786 2012, ApJL, 758, L38, doi: 10.1088/2041-8205/758/2/L38

787 Orozco Suárez, D., Bellot Rubio, L. R., del Toro Iniesta,

788 J. C., et al. 2007, ApJL, 670, L61, doi: 10.1086/524139

789 Parker, E. N. 1963, ApJ, 138, 552, doi: 10.1086/147663

790 Petrovay, K. 1994, in Solar Surface Magnetism, ed. R. J.

791 Rutten \& C. J. Schrijver (Dordrecht: Springer

792 Netherlands), 415-440,

793 doi: $10.1007 / 978-94-011-1188-1 \_35$

794 Rajaguru, S. P., Sangeetha, C. R., \& Tripathi, D. 2019,

795 ApJ, 871, 155, doi: 10.3847/1538-4357/aaf883

796 Rast, M. P. 2003, ApJ, 597, 1200, doi: 10.1086/381221

797 Requerey, I. S., Cobo, B. R., Gošić, M., \& Bellot Rubio,

798 L. R. 2018, A\&A, 610, A84,

799 doi: $10.1051 / 0004-6361 / 201731842$

800 Rieutord, M., Rincon, F., \& Roudier, T. 2012, in EAS

801 Publications Series, Vol. 55, EAS Publications Series, ed.

802 M. Faurobert, C. Fang, \& T. Corbard, 5-13,

803 doi: 10.1051/eas/1255001

804 Rouppe van der Voort, L. H. M., Rutten, R. J., \& Vissers,

805 G. J. M. 2016, A\&A, 592, A100,

806 doi: $10.1051 / 0004-6361 / 201628889$ 
807 Schuck, P. W. 2006, ApJ, 646, 1358, doi: 10.1086/505015

808 -. 2008, ApJ, 683, 1134, doi: 10.1086/589434

809 Schüssler, M. 1986, in Small Scale Magnetic Flux

810

Concentrations in the Solar Photosphere, ed. W. Deinzer,

M. Knölker, \& H. H. Voigt, 103

812 Shelyag, S., Keys, P., Mathioudakis, M., \& Keenan, F. P.

${ }_{813}$ 2011, A\&A, 526, A5, doi: 10.1051/0004-6361/201015645

814 Simon, G. W., Title, A. M., \& Weiss, N. O. 2001, ApJ, 561,

815 427, doi: 10.1086/322243

816 Simon, G. W., \& Weiss, N. O. 1989, ApJ, 345, 1060,

817 doi: $10.1086 / 167976$

818 Stangalini, M., Giannattasio, F., \& Jafarzadeh, S. 2015,

819 A\&A, 577, A17, doi: 10.1051/0004-6361/201425273

820 Stenflo, J. O. 1982, SoPh, 80, 209, doi: 10.1007/BF00147969

821 - 2013a, A\&A, 555, A132,

822 doi: $10.1051 / 0004-6361 / 201321608$
${ }_{823}$ —. 2013b, A\&A Rv, 21, 66, doi: 10.1007/s00159-013-0066-3

${ }_{824}$ Trujillo Bueno, J., Shchukina, N., \& Asensio Ramos, A.

825 2004, Nature, 430, 326, doi: 10.1038/nature02669

${ }_{826}$ Tsuneta, S., Ichimoto, K., Katsukawa, Y., et al. 2008,

${ }_{827}$ SoPh, 249, 167, doi: 10.1007/s11207-008-9174-z

${ }_{828}$ Viticchié, B., Del Moro, D., \& Berrilli, F. 2006, ApJ, 652,

829 1734, doi: 10.1086/508332

830 Weiss, N. 2001, Astronomy and Geophysics, 42, 3.10,

831 doi: 10.1046/j.1468-4004.2001.42310.x

832 Welsch, B. T., Fisher, G. H., Abbett, W. P., \& Regnier, S.

833 2004, ApJ, 610, 1148, doi: 10.1086/421767

${ }_{834}$ Welsch, B. T., Kusano, K., Yamamoto, T. T., \& Muglach,

835 K. 2012, ApJ, 747, 130,

${ }_{836}$ doi: $10.1088 / 0004-637 \mathrm{X} / 747 / 2 / 130$

837 Wien, W. 1916, Annalen der Physik, 354, 842,

838 doi: 10.1002/andp.19163540704 